Revue

Revue de l'histoire des religions

del'histoire des religions

$1 \mid 2017$

Varia

\title{
Anna Lisa ScHINo, Battaglie libertine. La Vita e le opere di Gabriel Naudé
}

Firenze, Le Lettere (« Giornale critico della filosofia italiana », 29), 2015

Jean-Pierre Cavaillé

\section{OpenEdition}

Journals

Édition électronique

URL : http://journals.openedition.org/rhr/8709

DOI : ERREUR PDO dans /localdata/www-bin/Core/Core/Db/Db.class.php L.34 : SQLSTATE[HYO00]

[2006] MySQL server has gone away

ISSN : 2105-2573

Éditeur

Armand Colin

Édition imprimée

Date de publication : 1 mars 2017

Pagination : 185-188

ISBN : 978-2-200-93125-4

ISSN : 0035-1423

\section{Référence électronique}

Jean-Pierre Cavaillé, « Anna Lisa Schino, Battaglie libertine. La Vita e le opere di Gabriel Naudé », Revue de I'histoire des religions [En ligne], 1 | 2017, mis en ligne le 24 mars 2017, consulté le 08 janvier 2021. URL : http://journals.openedition.org/rhr/8709 ; DOI : https://doi.org/ERREUR PDO dans /localdata/ www-bin/Core/Core/Db/Db.class.php L.34 : SQLSTATE[HY000] [2006] MySQL server has gone away

Ce document a été généré automatiquement le 8 janvier 2021

Tous droits réservés 


\section{Anna Lisa ScHINO, Battaglie libertine. La Vita e le opere di Gabriel Naudé}

Firenze, Le Lettere (« Giornale critico della filosofia italiana », 29), 2015

Jean-Pierre Cavaillé

\section{RÉFÉRENCE}

Anna Lisa SchINO, Battaglie libertine. La Vita e le opere di Gabriel Naudé, Firenze, Le Lettere («Giornale critico della filosofia italiana », 29), 2015, 22,4 cm, 304 p., $35 €$, ISBN 978-88-6087-829-8.

1 Ces Batailles libertines. Vie et œuvres de Gabriel Naudé, constituent sans nul doute un ouvrage important sur le fameux érudit bibliothécaire, considéré comme l'un des représentants majeurs de ce qu'il est convenu d'appeler, depuis René Pintard, le «libertinage érudit ». Il s'agit d'ailleurs d'un livre lui-même très érudit, tout à fait représentatif de l'histoire des idées à l'italienne, d'une grande rigueur descriptive (par exemple dans le comparatisme serré des auteurs dont les relations sont explicites), historiographique (multiples références aux collègues) et philologique (ici, il n'est pas question de traduire une citation française ou latine, le lecteur visé est nécessairement polyglotte!). L'auteure ne renonce pas à l'usage du concept de libertinage, mais elle préfère parler $d$ '«opérations intellectuelles libertines visant à s'affranchir de la religion et à transformer le point de vue du lecteur » (p. 15).

2 L'ouvrage contient d'abord une biographie intellectuelle actualisée et synthétique qui a le mérite, entre autres choses, d'insister sur l'importance de la formation médicale de Naudé, qui imprègne considérablement son œuvre (au-delà des Questions iatrophilologiques que A. L. Schino est l'une des seules à avoir travaillées) et sa manière d'appréhender le réel. Après René Pintard ou Lorenzo Bianchi, elle insiste sur l'importance déterminante de l'expérience italienne: la rencontre admirative de Cremonini, les relations compliquées avec Campanella, le réseau de solidarité autour de Galilée, etc. Le second chapitre est justement consacré au programme méthodologique 
de Naudé, dominé par une conviction énoncée dans une lettre à Jacques Dupuy : «J'ai conclu il y a longtemps que la plupart des choses merveilleuses ne seraient pas tant estimées si on pouvait connaître les impostures ou le peu de jugement de ceux qui les rapportent $»$.

Pour Naudé, résume Schino, « les premières causes de l'erreur sont l'ignorance et la merveille, la peur et l'habitude»(p.100). Ce bibliographe philosophe est résolument rationaliste, mais la raison qu'il promeut est «dépouillée de tout intellectualisme, héritière d'une tradition empirique, imprégnée d'aristotélisme, mais lu dans le sillage des Padouans: une raison pragmatique et utilitariste qui trouve son emploi dans l'enquête et dans le jugement condamnant les erreurs, faussetés et superstitions " (p.98). Il s'agit donc d'un rationalisme antidogmatique, qui met en avant un usage critique et analytique de la raison, fuyant toute visée systématique, mais pour autant non sceptique : Naudé ne suspend pas son jugement ; il rejette les faux savoirs, ceux qui prétendent porter sur des objets surnaturels inaccessibles à la raison humaine, au profit des vérités factuelles, telles que l'histoire naturelle, civile ou ecclésiastique peut les établir. Dans sa promotion de l'histoire il suit en effet les définitions et la tripartition bodinienne établie dans la Methodus. L'histoire ecclésiastique, bien sûr, n'est pas l'histoire sacrée, assimilée au mythe et expulsée comme telle des disciplines historiques dignes de ce nom. L'histoire politique et civile, quant à elle, possède une finalité éminemment pratique : associée à l'expérience directe, elle joue le plus grand rôle dans l'acquisition de la prudence, entendue comme "vertu de l'intellect pratique et savoir voué à la vie active ", elle sert ainsi à former et guider les esprits d'exception affranchis des impostures, maîtres d'eux-mêmes et capables de grandes choses (Schino cite à ce propos un passage mal connu mais lumineux du Syntagma de studio liberali, p. 283).

4 Naudé s'emploie ainsi, en tous ses travaux, à rapporter intégralement les phénomènes considérés comme prodigieux ou surnaturels à l'ordre naturel, suivant en cela les philosophes italiens «modernes » qu'il exploite le plus - Pomponazzi et Cardan -, à la différence fondamentale près qu'il ne se réfère jamais positivement à des causes "méta-humaines et métahistoriques" (en particulier les conjonctions astrales), l'explication étant toujours recherchée dans le contexte social et politique des individus et des peuples (p. 107).

5 La première application de cette méthode fut, comme le montre Schino, l'enquête que Naudé consacra à l'apparition des Rose-Croix, véritable déconstruction du phénomène qui réduit celui-ci au statut de "fable ». C'est la même démarche qui anime ses autres ouvrages, et en particulier l'Apologie pour tous les grands hommes qui ont été accusés de magie, l'œuvre de Naudé à laquelle l'auteure prête la plus grande attention, lui consacrant plusieurs chapitres. Ses adversaires majeurs y sont les démonologues et les apologètes qui, comme Martin Del Rio ou Pierre le Loyer (j'ajouterai Henri de Montagu pour les premiers chapitres: Daemonis mimica in magiae progressu tum in sectis errorum quorum author est [...] Henrici a Monteacuto, [...] elucubratiunculis, Paris, Claude Rigaud, 1612) sont si prompts à déceler l'action du diable dans les affaires humaines et, en particulier, dans les spéculations intellectuelles s'écartant un tant soit peu des dogmes établis. Les pratiques magiques, angéliques ou démoniaques, sont ainsi ramenées strictement à la magie naturelle ou à la simple imposture, et la présence même des esprits est radicalement mise en question. Schino accompagne Naudé pas à pas dans cette entreprise de démontage des fausses accusations portées à une série de 
personnages fameux (Zoroastre, Orphée, Pythagore, Platon, Porphyre, Jamblique, Proclus, Apulée, al-Kindi, Geber, Thébit [Thābit ibn Qurra], Raimond Lulle, Arnauld de Villeneuve, Pietro d'Abano, Roger Bacon, Robert Grossetête, Albert le Grand, Trithème, Agrippa de Nettesheim, Paracelse). Dans cette série d'études, fondées sur le recoupement et l'évaluation des sources, Schino, voit à juste titre les prodromes de ce qui deviendra le Dictionnaire historique et critique de Bayle. La relation est aussi frappante avec les cahiers de Guy Patin, établis à partir de conversations avec Naudé lui-même et d'autres érudits. Les Naudeana, souvent cités par l'auteure, sont tirés de ces cahiers, autrement plus riches pour la reconstitution de la vie et de la culture italienne de Naudé. Voir en particulier, le manuscrit de Vienne (Stadtbibliothek, codex 7071 Hohendorf 133). La partie du manuscrit où Naudé parle en première personne - sous la copie de Patin - est d'ailleurs problématique quant à la position de Naudé en matière de religion, car il y affirme un fidéisme catholique qui n'est autre que celui de Patin luimême (qui tient la plume !).

6 Naudé aborde également dans cet ouvrage (ainsi que dans son Jugement de Cardan) la question des démons familiers, pour en donner une explication naturaliste et même "résolument matérialiste, épurant la théorie de Ficin de tout élément relevant de l'astrologie ou de la magie spirituelle et la reconduisant à une théorie médicale du tempérament» (p.176), refusant avec énergie, de manière couverte ou, dès qu'il le peut, ouverte, d'accorder un quelconque crédit aux phénomènes prétendus de sorcellerie et de possession : il est visiblement en accord avec Averroès dont il dit dans l'Apologie qu'il "n'a jamais cru qu'il y eût des Diables». Schino fait justement remarquer que la défense de Cardan (assortie d'ailleurs de critiques mordantes) montre à quel point Naudé est détaché de la religion; car dans son Judicium Cardani, c'est bien l'entreprise même de la réduction naturaliste de la naissance du christianisme qu'il défend.

7 Dans sa Question iatrophilologique sur le destin et l'issue fatale de la vie, texte passionnant dont Schino a donné une réédition (Quaestio iatrophilologica de fato et fatali vitae termino, in Joh. Beverovicii Epistolica quaestio de vitae termino, fatali an mobili? Cum doctorum responsis, Lugduni Batavorum, ex Officina Ioannis Maire, 1639, ristampa anagrafica, edizione Anna Lisa Schino, Lecce, Conte Editore, 1995), Naudé procède à une démystification du fatum et à une critique du fatalisme d'une grande proximité avec le De Fato d'Alexandre d'Aphrodise qui exploite Aristote contre le déterminisme stoïcien ( le destin se trouve dans ce qui arrive par nature, de sorte que destin et nature sont la même chose »). Or, il est possible d'agir sur la nature, certes dans des limites étroites, et c'est bien ce à quoi s'emploie la médecine, pour ce qui est de la vie humaine. Prenant pour guide l'aristotélisme alexandriste, Naudé est amené à affirmer son net désaccord avec Pomponazzi, plus authentiquement averroïste, dont pourtant l'importance reste grande pour lui. De toute façon, l'aristotélisme de Naudé n'est pas dogmatique, il est d'abord un guide et un modèle pour la formation de «l'habitus mental de chercheur ». Schino consacre enfin un chapitre assez rapide à ce qui est le mieux connu chez son auteur : ses écrits politiques et en particulier son ouvrage sur les coups d'État, dont elle rapporte qu'il « est moins caractérisé par l'immoralité, qui est une donnée constante du pouvoir politique, que par le risque, le secret, l'imprévisibilité et le caractère péremptoire des choix et des comportements » (p. 262).

Un ouvrage donc, on le voit, très riche et dont les études sur le « libertinage » devront désormais tenir compte. En guise de critique, je me contenterai de regretter que la 
querelle fleuve qui occupa Naudé tant d'années, concernant l'identification de Thomas a Kempis comme auteur de l'Imitation du Christ soit traitée de manière si rapide et cavalière («il n'était probablement pas la personne juste pour décider la question », p. 77). Le dossier pourtant mériterait sans aucun doute une étude approfondie, étant donné l'ampleur, la durée et la virulence d'une controverse encore ouverte aujourd'hui, d'autant plus que celle-ci présente un magnifique exemple de dénonciation d'imposture (en l'occurrence une falsification de document). Le fait que Naudé, par-delà les siècles, continue d'être insulté mais non réfuté par ceux qui s'acharnent à attribuer l'Imitation à Gerson ou à Gersen devrait pourtant suffire à susciter l'attention des chercheurs - faute de toute autre étude, je me permets de renvoyer à mon article: "Une chose bien remarquable et importante à la République des Lettres, Gabriel Naudé et l'attribution frauduleuse de l'Imitation de Jésus-Christ à Jean Gersen ", Comparaisons, raisons, raisons d'État. Les politiques de la république des lettres au tournant du XVII siècle, sous la dir. d'Armelle Lefebvre, Munich, R. Oldenbourg Verlag, 2010, p. 152-177.

\section{AUTEURS}

\section{JEAN-PIERRE CAVAILLÉ}

École des Hautes Études en Sciences Sociales, Toulouse. 